\title{
Varikosis der VSM: Höhere Rekanalisationsrate nach MOCA als nach ELVA und RFA
}

Vähäao S et al. Randomized clinical trial of mechanochemical and endovenous thermal ablation of great saphenous varicose veins. BJS 2019; 106: 548-554

Die Behandlung variköser Venen hat sich erheblich verändert; inzwischen sind verschiedene minimalinvasive Methoden verfügbar. Die endovenöse Thermoablation mit Laser (EVLA) oder Radiofrequenz (RFA) erreichen langfristige Okklusionsraten von > $90 \%$ und scheinen den Okklusionsraten der Schaum-Sklerotherapie überlegen. Die Studie verglich die mechanochemische Ablation (MOCA) mit der EVLA und RFA.

Die MOCA erhitzt nicht die Vene oder das umgebende Gewebe und erfordert daher keine Tumeszenzanästhesie. Zum Outcome gibt es bislang sehr wenige Daten. Von 2014 bis 2015 wurden alle Patienten, die sich in einer gefäßchirurgischen Klinik wegen variköser Venen vorstellten, auf eine Insuffizienz der V. saphena magna (VSM) untersucht. Für die Studie wurden Patienten im Alter von 20-75 Jahren aufgenommen

- mit einem klinischen Stadium C2-C4,

- mit im Ultraschall festgestelltem Reflux in die VSM sowie

- mit einem mittleren Durchmesser der VSM im Oberschenkel von 5-12 mm.

132 wurden randomisiert für MOCA, ELVA oder RFA. Letztendlich in die Studie aufgenommen wurden 125, von denen 117 das
1-Jahres-Follow-up vollendeten. Von diesen erhielten 55 eine Therapie mit MOCA, 33 eine mit EVLA und 29 eine mit RFA. In allen Gruppen wurden begleitende Phlebektomien durchgeführt. Primäres Outcome war die Okklusionsrate der VSM nach 1 Jahr. Sekundäre Outcomes waren

- die krankheitsspezifische Lebensqualität,

- Schmerzen während und nach der Behandlung,

- die Dauer der Krankschreibung,

- die erforderliche Schmerzmittelmedikation während und nach der Behandlung,

- die Okklusionsrate nach 30 Tagen sowie

- Komplikationen.

Nach 1 Jahr waren die behandelten Abschnitte der VSM bei allen mit EVLA oder RFA behandelten Patienten komplett okkludiert (Okklusionsrate: $100 \%$ ). In der MOCA-Gruppe war dies bei 45/55 Behandelten der Fall (Okklusionsrate $82 \%$ ). In der MOCA-Gruppe trat bei 10 Personen im behandelten Abschnitt der VSM eine Rekanalisation auf, bei 7 Personen im proximalen Abschnitt. In diesem Segment bestand eine starke Assoziation zwischen dem präoperativen Durchmesser der VSM und der Rekanalisationsrate. Die auf der VAS-Skala angegebenen Schmerz-Scores während und bis zu 1 Woche nach der Intervention waren ähnlich. Die Dauer der Krankschreibung unterschied sich in den Gruppen nicht signifikant. Sie betrug nach MOCA im Mittel 4,3 Tage, nach EVLA 5,3 Tage und nach RFA 4,7 Tage. Auch der Umfang der Schmerzmedikation nach dem Eingriff unterschied sich zwischen den Gruppen nicht. Die angegebene Lebensqualität war zwischen den Gruppen ähnlich.

\section{FAZIT}

Ihre Ergebnisse zeigen, so die Autoren, dass die Behandlung mit MOCA in Kombination mit Phlebektomien nicht weniger schmerzhaft ist als die Thermoablation. Da die begleitenden Phlebektomien die Schmerz-Scores beeinflussten, sind diese möglichen Unterschiede zwischen den Ablationstechniken selbst nicht zu entnehmen. Wegen der Sedierung mit Propofol während der Thermoablation war eine verlässliche Registrierung der Schmerz-Scores nicht möglich.

Dr. Gabriele Dobler, Berlin 